duct and machine-assisted disinfection using hot water $\left(170^{\circ} \mathrm{F}\right.$ for 30 minutes). Both types of machines had a wash cycle built into the process, followed by the disinfection cycle. The chemically-assisted machine also had a rinse cycle. Our study differed from previously published studies in that we inoculated the tubing with a known inoculum of Pseudomonas species and Acinetobacter species. We found that in our study the machine-assisted chemical disinfection was more efficient in killing the organism we had introduced than the hot water disinfection alone.

Our study will be published shortly in the Journal of Hospital Infection (British).

Inge Gurevich, R.N.
Patricia Tafuro, R.N.
Infection Control Practitioners
Nassau Hospital
Mineola, L.I., New York

\section{Sterilization of Surgical Instruments}

\section{To the Editor:}

A question regarding proper sterilization of surgical instruments has arisen for us, and we are hoping you may be able to help us with the solution. For some time we have been aware that instruments must be left unclamped and open in surgical packs to enable the autoclave steam to penetrate to all surfaces in order to assure sterility.
The surgery crew is afraid to do this with sharp instruments such as towel clips and tenaculums, however, because of the danger to the staff in opening the packs. Most of the staff claim they have had unfortunate accidents of this nature in the past. We know that hooking them into a towel is not a suitable solution because of the potential problems with "holy" surgical linen.

We are hoping you have encountered dilemmas such as this before and can "shed some light" on our problem.

Annette Rhodes, R.N. Infection Control Coordinator Barton Memorial Hospital South Lake Tahoe, California

The preceding letter was referred to Carole Van Antwerpen, R.N., and Peter A. Gross, M.D., for a reply.

Safe packaging for sterilization is a common problem with sharp instruments such as towel clips and tenaculums.

In our operating room, after the cleaning process towel clips and tenaculums are autoclaved in baskets in the unlocked position. We define unlocked as keeping the edges touching while the instrument is not locked completely. In this position you can imagine that the tips of the towel clips or tenaculums are just touching or are slightly separated. The use of this technique has virtually eliminated unfortunate accidents.
In our central sterile processing area towel clips and tenaculums are usually packaged in procedure trays or heatsealed pouches. Again the unlocked position rather than the completely open position is preferred. For the trays, a towel is placed over the sharp objects to prevent unnecessary punc. ture wounds when opening the trays. For the peel-back pouches, a square piece of gauze $(3 \times 3$ or $4 \times 4)$ is placed over the sharp points. This method will prevent the tips from piercing the pouch. So this method will not only keep the instrument sterile while it is in the pouch but, also prevent puncture wounds when the instrument is being removed from the pouch. Whatever preventive measures are used, inservice education on a continuing basis is necessary to minimize the hazards of sharp instruments for the staff.

\section{REFERENCES}

1. Perkins J: Processing of surgical instruments, in Principles and Methods of Sterilization in Heallh Sciences, ed 2. Springfield, Illinois, Charles C Thomas Pub, 1976, p 257.

2. Principles and procedures of asepsis, in Rhodes MJ, Gruendeman BJ, Ballinger W (eds): Alexander's Care of the Patient in Surgery, ed 6. St Louis, CV Mosby Co, 1978, p 67 .

Carole Van Antwerpen, R.N. Nurse Epidemiologist Peter A. Gross, M.D. Director, Department of Internal Medicine Professor of Medicine, New Jersey Medical School University of Medicine and Dentistry of New Jersey Hackensack, New Jersey 


\section{Many Healthcare handwash- ing products contain proven antimicrobial ingredients. However, Calgon's new Acute- Kare Healthcare Personnel Handwash, with 1\% PCMX, has been shown to be signif- \\ icantly superior in FINAL \\ FORMULATION, to current widely used products in rate and completeness of bacterial kill. And, its emollient- enriched formula is gentle, even after repeated use.}

CALGON ACUTE-KARE ${ }^{\mathrm{m}}$

The Most Important Ingredient In Your Healthcare Personnel Handwash Is Not Always The Ingredients...

*As defined in the FDA Panel 1 monograph. Federal Register 1/6/78.

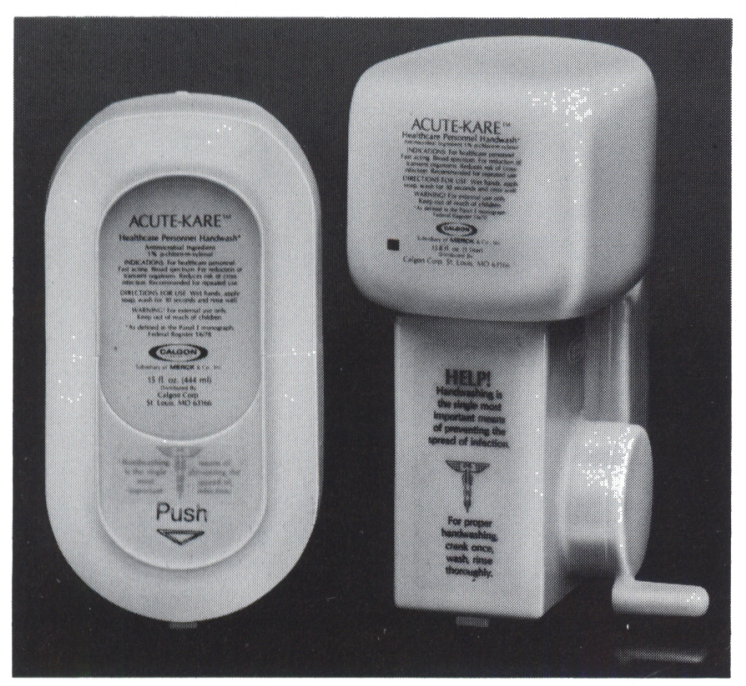

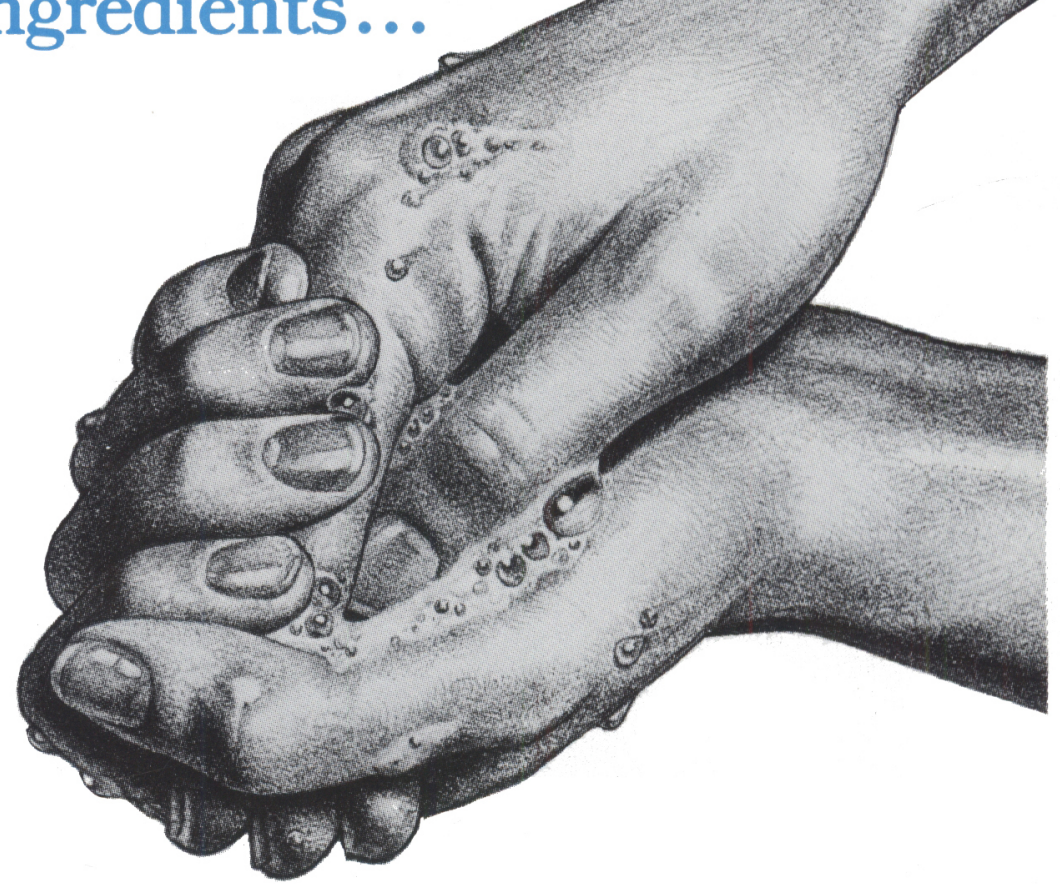

\section{Antimicrobial Protection You Can Count On...}

- Acute-Kare is recommended for high use frequency locations where antimicrobial insurance against contamination from transient organisms is required or desirable.

- Contains 1\% PCMX (Para-chloro-meta-xylenol), a broad spectrum antimicrobial ingredient recognized as safe to use in topically applied products.

- Broad spectrum-activity against gram negative and gram positive bacteria and yeast.

- Residual Activity-Handwashing tests following an FDA approved protocol, show a significant reduction in inoculated microorganisms versus a control after 25 wash sequences.

\section{With Gentleness You Deserve...}

- Acute-Kare is non-irritating, even after repeated use.

- Acute-Kare contains unique skin conditioners and emollients.

- Acute-Kare has a pH balanced formula which is compatible with the skin's natural $\mathrm{pH}$.

\section{Call Your Calgon Healthcare Sales Specialist For A FREE Demonstration And Test.}

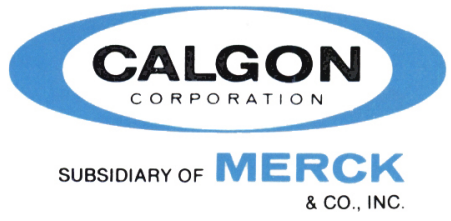


